\title{
Endoscopic ultrasound for the characterization and staging of rectal cancer. Current state of the method. Technological advances and perspectives.
}

\author{
Mariana M. Gersak ${ }^{1}$, Radu Badea ${ }^{2}$, Florin Graur ${ }^{3}$, Nadim Al Hajjar ${ }^{3}$, Luminita Furcea ${ }^{3}$, \\ Sorin M. Dudea ${ }^{1}$
}

${ }^{1}$ Department of Radiology Cluj County Clinical Emergency Hospital, "Iuliu Hatieganu" University of Medicine and Pharmacy, ${ }^{2}$ Ultrasound Imaging Laboratory, Department of Imaging and Radiology, "Octavian Fodor" Regional Institute of Gastroenterology and Hepatology, "Iuliu Hatieganu" University of Medicine and Pharmacy ${ }^{3} 3^{\text {rd }}$ Surgical Clinic, "Octavian Fodor" Regional Institute of Gastroenterology and Hepatology Cluj-Napoca, Romania

\footnotetext{
Abstract

Endoscopic ultrasound is the most accurate type of examination for the assessment of rectal tumors. Over the years, the method has advanced from gray-scale examination to intravenous contrast media administration and to different types of elastography. The multimodal approach of tumors (transrectal, transvaginal) is adapted to each case. 3D ultrasound is useful for spatial representation and precise measurement of tumor formations, using CT/MR image reconstruction; color elastography is useful for tumor characterization and staging; endoscopic ultrasound using intravenous contrast agents can help study the amount of contrast agent targeted at the level of the tumor formations and contrast wash-in/wash-out time, based on the curves displayed on the device. The transvaginal approach often allows better visualization of the tumor than the transrectal approach. Performing the procedure with the rectal ampulla distended with contrast agent may be seen as an optimization of the examination methodology. All these aspects are additional methods for gray-scale endoscopic ultrasound, capable of increasing diagnostic accuracy. This paper aims at reviewing the progress of transrectal and transvaginal ultrasound, generically called endoscopic ultrasound, for rectal tumor diagnosis and staging, with emphasis on the current state of the method and its development trends.

Keywords: transrectal ultrasound, CEUS, color elastography, rectal tumors
}

\section{Introduction}

Colorectal cancer is the third most common malignant neoplasm, being responsible for approximately $8-9 \%$ of deaths, as shown by official statistics in the USA for 2014 [1].

Pathology and hereditary factors that increase the risk of colorectal cancer play a very important role. Family history of colorectal cancer, polyps, or a history of ulcerative colitis, Crohn's disease, Lynch syndrome, familial

Received 15.04.2015 Accepted 02.05.2015

Med Ultrason

2015, Vol. 17, No 2, 227-234

Corresponding author: Radu Badea

$3^{\text {rd }}$ Medical Clinic

19-21 Croitorilor Street

400162 Cluj-Napoca, Romania

E-mail: rbadea@umfcluj.ro adenomatous polyposis, and type 2 diabetes should be taken into account when discussing the risk of developing colorectal $[2,3]$. Histological over $90 \%$ of colorectal cancers are adenocarcinomas originating in the singlelayer epithelium of the mucosa. The degree of tumor differentiation is associated with tumor aggressiveness [3].

The final outcome of colorectal cancer therapy, assessed through the 5-year survival rate, is subject to the tumor stage of the neoplasm when detected [4]. Depending on rectal tumor extension, the presence of lymph nodes and metastases, patients may undergo immediate surgery with curative intent, neoadjuvant therapy followed by surgery, or chemotherapy-radiotherapy possibly followed by palliative surgery [5]. In clinical medicine, tumor staging based on digital rectal examination has been the basic method for many years [6]. In recent decades, this simple gesture, shown to be subjective and with relatively low sensitiv- 
ity, has been replaced by imaging procedures. Magnetic resonance imaging (MRI), computed tomography (CT), and endorectal ultrasound (US) are some of the imaging procedures accepted for rectal tumor staging $[6,7]$.

Researchers' concerns regarding transrectal US were focused on assessing prostate lesions [8]. The first rectal tumor recurrence was highlighted by transrectal approach in 1953 by Wild and Reid, who used the equipment employed for prostate assessment [9]. It was only later, in 1983 , that medical publications mentioned the transrectal ultrasound approach to rectal tumors $[10,11]$. Shortly after this, the ultrasonographic anatomy of the rectal wall was described. Thus, transrectal US (TRUS) can easily identify each layer of the rectal wall, corresponding to a total of five laminae, some interfaces and some real anatomical structures, all with hypo or hyperechoic aspect [12].

TRUS has rapidly expanded and overall results concerning rectal tumor staging have become encouraging, compared to digital rectal examination and CT examination with sensitivity (Se) $94 \%$, specificity (Sp) $87 \%$, positive predictive value (PPV) 97\%, negative predictive value (NPV) 78\% and overall accuracy (OA) 91\% [13]. Moreover, TRUS has also demonstrated its efficacy in identifying lymph nodes and local recurrence [14,15].

Preoperative US staging classification called uTNM $(\mathrm{u}=$ ultrasound, $\mathrm{TNM}=$ tumor, nodes, metastases $)$ was proposed in 1985 by Hildebrandt and Feifel, being rapidly assimilated in Europe [16-18]. Subsequently, the transvaginal approach of rectal tumors was developed, important for a better prominence of peritumoral lymph nodes, together with overall rectovaginal space examination and stenotic rectal tumor examination $[19,20]$.

\section{Ultrasound procedures}

Gray scale US (2D, 3D).

This is the basic procedure for the investigation. It has a morphological character, with very good image quality and better spatial resolution than CT or MRI. During tumor detection, TRUS must provide the following information: tumor morphology (proliferative, stenotic, infiltrative) and tumor size, tumor extension into the longitudinal muscle, penetration depth, infiltration of pelvic organs, position in relation to the anal orifice, complications such as abscesses and fistulae, the presence and characteristics of perirectal lymph nodes (shape, echogenicity, delimitation) and, finally, US staging [21]. Gray scale US can also be used for needle guidance of biopsies [22]. Three-dimensional (3D) examination, derived from two-dimensional US, is an additional application of certain devices, which allows the creation - by multiplanar reconstructions - of similar tumor formation images visi- ble to the naked eye. This application is mainly exploited in the field of obstetrics and gynecology [23], but it also has functions in other clinical areas. The method is useful for the accurate measurement of tumor volume and for the assessment of the relationship with the surrounding organs, especially for patients scheduled for surgery. A considerable advantage of 3D US is the rotation of the volume data procedure, as well as the volume sectioning procedure achieving a real virtual US endoscopy.

\section{Elastography}

This procedure is a step forward in the ultrasonographic assessment of rectal tumors. When elastography entered the clinic, US has become an "extension" of the clinical sense, strengthening and confirming the final diagnosis. Depending on the equipment and how tissue elasticity is measured, elastography is divided into color or strain elastography imaging (SEI) and shear wave imaging (Acoustic Radiation Force Impulse imaging (ARFI) and Shear Wave Elasticity Imaging (SWEI)) [24]. ARFI and SWEI are quantitative methods, with well-known clinical applications in the assessment of the liver, but also of other organs. No data have been published so far concerning their use in the assessment of rectal tumors. Real-time color elastography (SEI) is the most accessible of all types of elastography, as it is a qualitative method that indicates tissue stiffness on color maps. Depending on the transducer, equipment and area of interest, the intermittent external compression on the transducer may be required, in order to obtain a valid image. Tissue elasticity is represented on a red to blue color scale. This type of elastography allows the comparison of tissue stiffness degrees of connected or neighboring tissues (tissue tension variation or compression ratio - "strain ratio" - SR) [24]. For an accurate calculation of SR, the two regions of interest (ROI) should be positioned at a similar vertical distance from the transducer [25]. Endoscopic color elastography is a real-time method that requires small amplitude intermittent compression in order to obtain a valid image [26]. As the procedure is examiner-dependent, the screen has a quality indicator which needs to be reached for the investigation to be considered correct. Color elastography can only be interpreted after obtaining a stable and valid color map from a qualitative point of view, constant in terms of color allocation. Thus, for $\geq$ $80 \%$ of the images, the equipment must allocate the same color consistently. Subsequently, the tumor formation can be characterized from a semiquantitative point of view using the strain ratio (SR), which is the elasticity ratio between two tissues elected by manually determining the ROI: one with a known elasticity, and the other with unknown, undetermined elasticity. For the most accurate calculation of the SR, the two ROI should be placed at 
the same distance from the transducer and should have similar size [25]. Color elastography has demonstrated its applicability and usefulness in multiple clinical areas [27], with promising results in TRUS as well. There is hope that the method would help for a better rectal tumor staging and differentiation between adenomas and adenocarcinomas [28]. To identify rectal tumor formations, Waage et al have used the Hitachi equipment with a micro-convex endorectal transducer (5-10 MHz), rigid, with 360-degree US scanning [26]. The intermittent compression of rectal formations was achieved using a water balloon which completely covers the transducer, intermittent compression being reproduced by inflating and deflating the balloon. [26] A similar result may be obtained with a conventional transducer, with a 180 -degree US scanning feature, wrapped in a lubricated condom, applying compression directly on the lesion.

\section{Doppler US}

This method allows the assessment of the flow rate using the frequency variation principle of an ultrasonic beam reflected from a 'target' in motion. The spectral method provides information about flow characteristics, flow direction and flow type (arterial or venous). The color-coded method ( $\mathrm{CFI}=$ color flow imaging) is a representation on a preset color scale (usually red and blue) of the vectors related to red blood cell groups found in the blood structure. Doppler US is a valuable adjuvant to tumor investigation.

\section{Contrast-enhanced US (CEUS)}

This method, based on the intravenous administration of contrast agents, was introduced into clinical practice in the 90s. There are numerous studies in the literature proving the additional diagnostic value of this procedure. However, there are few published studies regarding the assessment of rectal tumors, possibly due to the fact that TRUS has only included this harmonic imaging method for a short period of time. CEUS examination is activated by the push of a button, which concomitantly triggers both the two-dimensional and the harmonic image. Before intravenous contrast medium administration, the mechanical index (MI) is set to a low value $(0.06-0.12)$ and the single US wave is placed deep into the area of interest. This is when the clock can be activated simultaneously with bolus intravenous contrast medium administration. The recommended dosage is of approximately $0.025 \mathrm{ml} / \mathrm{kg}$. Immediately after the administration of the contrast medium, $5-10 \mathrm{ml}$ of $0.9 \%$ saline is injected. Within seconds after contrast administration, one can already notice the appearance of microbubbles in the area of interest [29-31]. The images must be stored for further analysis of loading and unloading curves (TIC $=$ time intensity curve). CEUS allows the manual selection of one or more ROI, indicating their graphical representation of the loading and unloading curves, concurrently. This may reveal an inconsistent intake of the contrast agent into two or more areas, such as tumor area and diseasefree area. Moreover, the US software allows the calculation of more parameters: the exact moment when the contrast agent becomes visible ( $\mathrm{AT}=$ arrival time), the time when the contrast agent reaches the highest concentration within the ROI (TTP = time to peak), the time of maximum intensity ( $\mathrm{PI}=$ peak intensity), the area under the curve (AUC), the loading and unloading time $(\mathrm{TIC}=$ time intensity curve), the ascending slope (AS) and echo intensity (EI) [29-31]. Due to the loading curves and the opportunity to measure these parameters, there is a very good interobserver agreement [29].

\section{Examination technique. Transducers}

Transrectal examinations are easy to perform, do not last long, and are well tolerated by the patient. Prior preparation includes enema, which should be performed approx. 30-60 minutes before the examination. Intrarectal water instillation can improve the depiction and accuracy of the procedure, differentiating between the rectal lumen and the rectal wall. This procedure, described since the $90 \mathrm{~s}$, generates rectum relaxation, helping to reduce artifacts induced by intrarectal food debris/gas collection, with the easy identification of normal structures and pathological lesions localized in this area $[20,32,33]$. It is a substitute for the first transrectal technique, where the mechanically rotating transducer was equipped with a balloon filled with degasified water. The patient is placed in the left lateral decubitus position, the knee-to-chest position. The transducer, inside in a lubricated condom, is inserted into the rectum to a depth of 10-12 cm. In case of pain, a local anesthetic gel can be applied on the surface of the transducer. Radial, longitudinal, and oblique sections are visualized. Transvaginal examinations require the patient to be placed in the supine position, the gynecological lithotomy position. This approach is well tolerated by patients. However, their status and preferences should be taken into account. The advantages are given by the good and constant visualization of the tumor.

The conventional use of the transducer for tumor staging is mechanical, with the US beam axis perpendicular to the transducer axis. The transducer has a frequency of 5-7 MHz. It is wrapped in a rubber bag which is expanded with $30-50 \mathrm{cc}$ of degasified water. The resulting image is circular, determined by the 360-degree rotation of the transducer, with adjustable speed. This equipment has been optimized for small size tumors. Current equipment is less "dedicated". The transducer used for transvaginal 
scan can also be used for transrectal exams. The US beam can be emitted in the axis or obliquely, at a 60-degree angle. The tip of the transducer can be flat or curved. There are devices that emit perpendicular US beams simultaneously or sequentially. Other dedicated devices enable simultaneous information gathering and tridimensional image reconstruction.

\section{Normal appearance}

TRUS and transvaginal US (TVUS) are the only diagnostic imaging techniques that allow identification of the layers of the rectal wall. From the inside (lumen) outwards are identified: the interface between the transducer and the mucosa (hyperechoic), the mucosa (hypoechoic), the submucosa (hyperechoic), the muscle (hypoechoic), and the serous layer (hyperechoic) (fig 1) [12]. The 3D investigation indicates the smooth or slightly uneven surface of the rectal wall, depending on the extent of mucosal folds. When performing Doppler US the perirectal vessels and the intramural vessels of the submucosal plexus have a linear aspect without any penetration of the rectal wall. A great number of perirectal tortuous vessels, which penetrate the rectal wall, are considered to be pathological [34-36].

During the investigation, the rectal wall displays medium stiffness, similar to that of the perirectal fat tissue. CEUS examinations performed 10-15 seconds after injection show a progressive increase in harmonic echoes, a phenomenon that is more obvious in the submucosa. After approx. 30 seconds, contrast discharge occurs, which is intermittent.

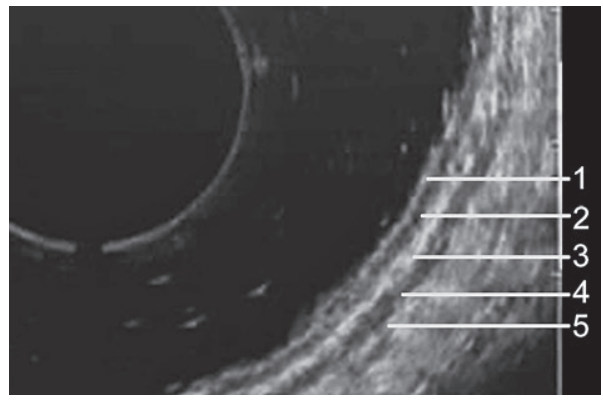

Fig 1. Normal rectal wall. Transrectal ultrasound. The wall layers can be observed, consisting of 5 different echogenic interfaces: 1 - the first layer (hyperechoic) represents the interface between water and the superficial mucosa; 2 - the second layer (hypoechoic) represents the mucosa; 3 - the third layer (hyperechoic) represents the submucosa; 4 - the fourth layer (hyoechoic) represents the muscularis propria; 5 - the fifth layer (hyperechoic) represents the interface between the serosa and perirectal fat

\section{Rectal tumors}

\section{Gray scale US (2D, 3D)}

The gray scale appearance of tumors, regardless of the histological structure, is that of localized transformation, with a variable structure and echogenicity of the rectal wall, which is measurable (fig 2). Concerning the assessment of rectal wall invasion, TRUS is superior in terms of accuracy and sensitivity (Se), specificity (Sp) and overall accuracy (OA) to CT, MRI and endorectal MRI ( $\mathrm{Se}=93 \%$, $78 \%, 86 \%$ and $89 \%$, respectively, $\mathrm{Sp}=78 \%, 63 \%, 77 \%$ and $79 \%$, respectively, $\mathrm{OA}=87 \%, 73 \%, 82 \%$ and $84 \%$ ) [37].

Rectal tumor staging is based on the internationally accepted TNM staging, adding the coefficient of error resulting from the specificity of the US image [4]. Due to this feature, the term uTNM is used, with the following correspondence with pathology: uT0 - no US image, as detected by current techniques or benign tumor, non-invasive lession; uT1 - invasive lesion with mucosal and submucosal confinement; uT2 - lesion confined to the muscularis propria - invasion of the second hypoechogenic layer; uT3 - lesions penetrating all rectal layers, the tumor invades the perirectal fat, without pelvic organ invasion; uT4 - tumor invasion into pelvic organs - prostate, vagina, uterus, cervix, bladder, etc.; uN0 - ultrasonographically undetectable adenopathy; uN1 - up to three ultrasonographically detectable tumor lymph nodes; uN2 - four or more ultrasonographically detectable tumor lymph nodes [4].

Endoscopic US is able to identify the circumferential resection margin of low rectal tumors confined to the anterior wall, with very good results (OA $87.5 \%$, negative predictive value - NPV-95.6\%) [38]. The method is also useful when distinguishing benign from malignant adenomas. Even in the $24 \%$ of the cases when rectal adenomas trigger a biopsy result that is negative for malignant cells, TRUS is able to correctly identify $81 \%$ of these cases as focal adenocarcinomas within adenomas [39]. TRUS is useful for the periodic assessment of low colorectal termino-terminal anastomosis, detecting local recurrences. The

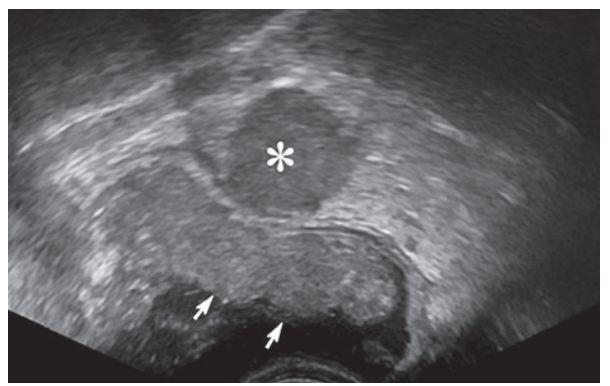

Fig 2. Gray scale transrectal ultrasound. Hypoechoic rectal tumor formation (arrows). Neoplastic adenopathy (asterisk). 
tridimensional aspect of rectal tumors is that of a protrusion or infiltration into the rectal wall (fig 3 ). Informations can be obtained in different incidence angles and can be "extracted" from the reconstructed volume. The US aspect of villous adenoma is that of a hypoechoic polypoid formation, inhomogenous, and well defined [30].

Regarding its role in increasing the diagnostic and staging value of 3D US, the accuracy of this method is similar or even superior to two-dimensional US (OA 69$84 \%$ for $2 \mathrm{D}$ TRUS and $78-88 \%$ for $3 \mathrm{D}$ TRUS $[40,41]$.

Malignant lymph nodes with peritumoral location have a hypoechoic aspect, round shape, $5-7 \mathrm{~mm}$ in size $[21,36,42,43]$. Reactive-inflammatory lymph nodes may sometimes have similar characteristics with the tumor [21]. A false lymph node positive image can be given by dilated perirectal vessels [21]. However, by sweeping the transducer in this area, the identification of a tubular trajectory and of vascular ramifications, as well as the presence of the vascular signal entirely covering the hypoechoic image, can easily explain this problem. The diagnostic value of TRUS is slightly inferior to that of transrectal MRI when assessing metastatic lymph nodes ( $\mathrm{Se}=71 \%$ vs. $82 \%, \mathrm{Sp}=76 \%$ vs. $83 \%, \mathrm{OA}=74 \%$, vs. $82 \%$ ) [37].

\section{Elastography}

Rectal tumors are stiffer than the tumor-free rectal wall and the perirectal fat tissue. An optimal differentiation between benign and malignant lesions was obtained at a cutoff value of the mean SR value of 1.25. Thus, malignant tumors, as compared to the tumor-free rectal wall and the perirectal fat tissue, have a mean SR value of $>1.25$. At this value, elastography differentiates between benign and malignant lesions much better than preoperative biopsy $(\mathrm{Se}=93 \%, \mathrm{Sp}=96 \%, \mathrm{OA}=94 \%$; preoperative biopsy: $\mathrm{Se}=69 \%, \mathrm{Sp}=87 \%, \mathrm{OA}=75 \%$ ) (fig 4) [26]. Rectal adenoma may be differentiated from adenocarcinoma using color elastography with additional gray scale TRUS value, being superior to MRI (TRUS: $\mathrm{Se}=96 \%, \mathrm{Sp}=62 \%$ and $\mathrm{OA}=90 \%$; elastography: Se with SR measurement: $\mathrm{Se}=96 \%, \mathrm{Sp}=86 \%$ and $\mathrm{OA}=94 \%$; $\mathrm{MRI}$ : $\mathrm{Se}=99 \%, \mathrm{Sp}=7 \%$ and $\mathrm{OA}=87 \%$ ) [28].

An interesting research work might be directed towards the role of color elastography in tumor staging. MRI cannot distinguish adenomas from early adenocarcinomas [44], even indicating a false-negative rate in pretherapeutic biopsy in $24 \%$ of cases [39]. Elastography combined with TRUS can differentiate between pT0 and pT1 tumor stages, the results being superior to MRI, including pretreatment biopsy. In advanced locoregional stages (T2 and T3 stages), elastography has not yet proved its usefulness in increasing diagnostic accuracy, taking into account the fact that TRUS has already shown a very good accuracy [28]. However, we must not forget that elastography examination is still insufficiently explored and, not least,

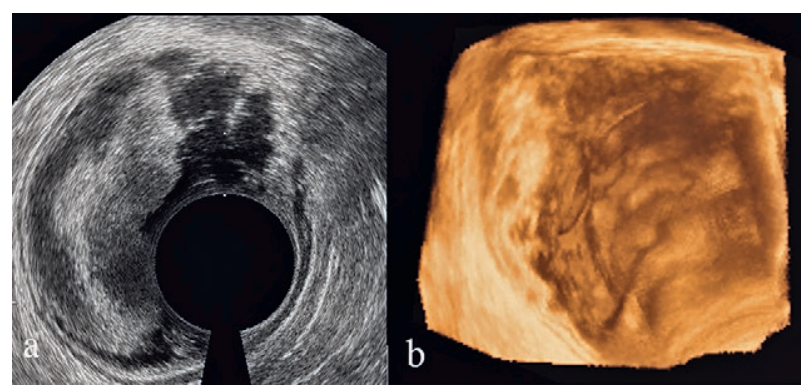

Fig 3. Infiltrative rectal lesion. Comparison between twodimensional gray scale ultrasound (a) and three-dimensional ultrasound (b).

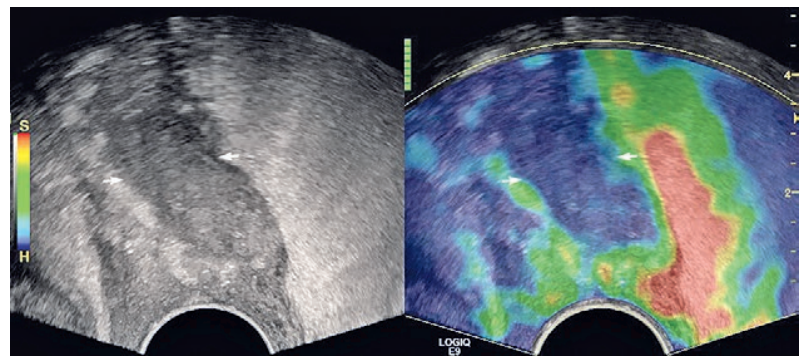

Fig 4. Hypoechoic tumor formation, stiff during elastography (arrows).

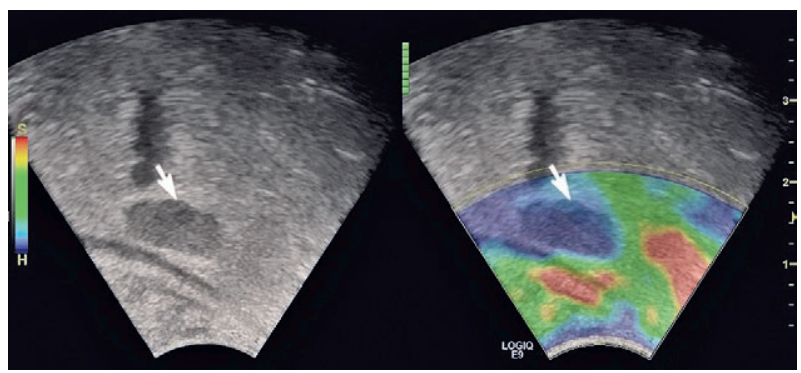

Fig 5. Neoplastic adenopathy (arrow) - gray scale ultrasound (left) and elastography - stiff tumor (right)

that it is not independent, but additional to TRUS. To our knowledge, until now, elastography assessment of lymph nodes has not been investigated. Still, at least in the case of bulky lymph nodes, it is expected that, based on the sensitivity of the current equipment, these lymph nodes should display increased stiffness (fig 5).

Elastography is limited by the presence of air and feces. These problems could be eliminated by performing a rigorous preparation and instilling a small amount of water rectally, immediately before the examination, with possible artifacts given by water. In large protrusive tumors, the direct contact between the water balloon, the tip of the transducer and the rectal wall may sometimes also be considered as a technical limitation [26]. The main problem of qualitative elastography is the interobserver variability due to uneven external compression [45]. However, with the development 
of elastographic methods (for examples the ones used for the assessment of thyroid nodules), interobserver variability proved to no longer be a drawback. Furthermore, the association of US examination with elastography has proven extremely useful for a better characterization of thyroid nodules [46-48]. Regarding endorectal elastography, there are still not enough studies to make firm statements. For now, the results are very promising in this regard [49].

\section{Doppler US}

Rectal tumor formations generally have a rich blood supply, but rather chaotic. Spatial distribution is uneven, with chaotic vessel orientation, displaced luminal trajectories, narrowing and expansion. There are many poorly irrigated areas associated with intratumoral necrosis. The vascular bed is dominated by arterial blood vessels (fig 6). The association of TRUS rectal tumor staging with Doppler US was studied in small groups of patients. After the research conducted by Sudakoff et al, Doppler US did not significantly improve the preoperative staging of rectal tumors, but it became useful in postoperative follow-up, enabling the differentiation between a possible residual tumor/tumor recurrence and postoperative scarring [34]. Another study defines the significant difference between T1/T2 stages and T3/T4 stages. When performing pulsed Doppler US, a value $>25 \mathrm{~cm} /$ second of peak systolic velocity (PSV) can differentiate between T3/T4 stages and $\mathrm{T} 1 / \mathrm{T} 2$ stages $(\mathrm{Se}=75 \%, \mathrm{Sp}=80 \%, \mathrm{OA}=70 \%)$. The values of the resistivity index (RI) were not significant when differentiating between these stages [36].

Color and pulsed Doppler US help differentiate between benign and malignant lymph nodes. Thus, a PSV

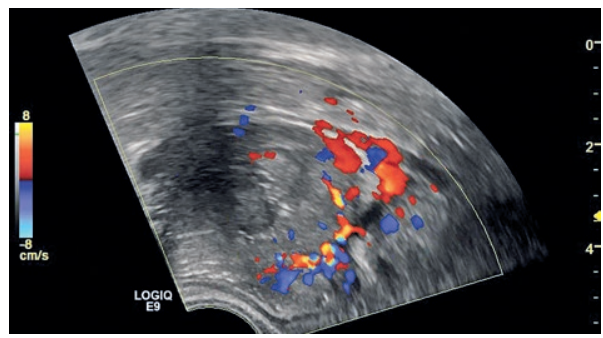

Fig 6. Color Doppler ultrasound. Hypervascular rectal lesion with penetrating intratumoral vessels value $>20 \mathrm{~cm} / \mathrm{second}$ and RI $>0.61$ indicate the malignancy of the lymph node $(\mathrm{Se}=100 \%, \mathrm{Sp}=62 \%, \mathrm{OA}=76 \%$; $\mathrm{Se}=71 \%, \mathrm{Sp}=84 \%, \mathrm{OA}=80 \%$ ). There is still the problem of differential diagnosis with reactive, inflammatory, hypervascularized lymph nodes, which can also show high PSV values, altering the specificity of the method $[36,50]$. In these cases, high RI values are suggestive of malignant lymph nodes, which can be explained by the fact that, within tumor formations, blood vessels are compressed, thus altering their resistance, while in case of inflammation, vessels are dilated, leading to a decrease in RI values [50].

CEUS

Unlike the normal rectal wall, adenocarcinomas have a rapid AT and TTP, while adenomas have a lower PI. Aggressive malignant tumors have a high degree of angiogenesis, with marked inhomogeneous imaging in 90\% of cases (fig 7). Large tumors can be characterized by low uptake or lack of uptake due to the presence of intratumoral necrosis [30]. Contrast discharge differs a lot in aspect, but in most cases, it is obvious during the portal venous phase. Rectal adenomas demonstrated their homogeneous uptake of contrast agent, tardily and with a lower intensity than the rectal wall, but large adenomas can also be characterized by an increased uptake [30].

Another purpose of the research was to assess tumor microvascular density, given that CEUS is superior to Doppler US in this field [51]. Wang et al studied the relationship between rectal tumor loading curve and microvascular density (MVD), with a positive correlation between EI (echo intensity) and MVD. Other correlations between the loading curve and MVD have not been identified, most likely due to the fact that the other parameters depend on tumor imaging speed and not on microvascular density [31]. There is a negative correlation between EI and T stage $(r=-0.295, p=0.007)$, which might suggest that tumor perfusion depends on T stage of [31], but this requires thorough research until it can firmly be concluded.

\section{General considerations. Limitations of US.}

Rectal tumor examination using the endoscopic approach must be clinically and multimodally integrated

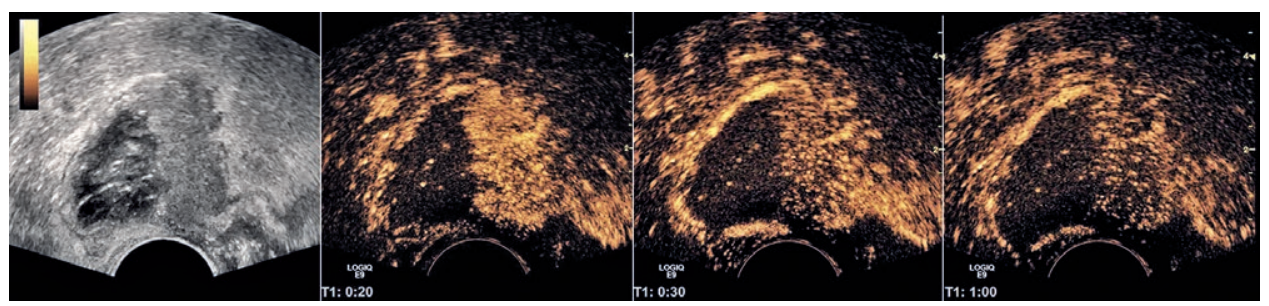

Fig 7. Villous adenoma - malignant; dynamic CEUS phases: early arterial phase ( 20 seconds) portal venous phase ( 30 seconds) and late arterial phase - complete washout within 60 seconds 
from a technological point of view. Clearly, the main aim of endoscopic exploration is tumor detection and tumor localization. TRUS or TVUS, as appropriate, can provide a great deal of morphological and vascular information designed to characterize the tumor and specify local invasion stage. Endoscopic US, like any other imaging technique, has several limitations: high rectal tumors are not accessible with the endocavitary transducer; stenotic circumferential neoplasm assessment (in men) prevents transducer sweeping; the posterior curve of the rectum may limit transducer accessibility for the optimal visualization of tumors located on the rear wall and lymph nodes from remote lymph node stations that can not be properly assessed, as they are not accessible. Currently, elastography and CEUS are the main lines of research, as well as additional TRUS methods. The main usefulness of elastography associated with TRUS lies in the identification of early neoplasms and the differentiation between early neoplasms and adenomas, where TRUS tends to indicate a lower stage and preoperative biopsy may be falsely negative. CEUS highlights tumor microcirculation better than Doppler US, but there are still efforts to valorize loading curve parameters and tumor staging. Research could further focus on the usefulness of elastography and CEUS in assessing rectal tumor response to neoadjuvant and adjuvant therapy, as well as on whether there is some correlation between the degrees of tumor differentiation based on US contrast loading curves.

\section{Conclusions}

Endoscopic US is a valuable method for the assessment, characterization and staging of rectal tumors. There are numerous US techniques: morphological (gray scale US), functional (elastography) and vascular (Doppler, CEUS). The combined use of these procedures allows an accurate assessment of rectal tumors before surgery, after radiotherapy/chemotherapy, and after surgery (for the detection of relapses).

\section{Conflict of interest: none}

Acknowledgment: We would like to thank Mrs. Zsoka Szasz for her help with image collection and systematization.

\section{References}

1. Siegel R, Ma J, Zou Z, Jemal A. Cancer statistics, 2014. CA Cancer J Clin 2014; 64: 9-29.

2. Schmoll HJ, Van Cutsem E, Stein A, et al. ESMO Consensus Guidelines for management of patients with colon and rectal cancer. A personalized approach to clinical decision making. Ann Oncol 2012; 23: 2479-2516.

3. Fleming M, Ravula S, Tatishchev SF, Wang HL. Colorectal carcinoma: Pathologic aspects. J Gastrointest Oncol 2012; 3: $153-173$.

4. How is colorectal cancer staged? Available from: http:// www.cancer.org/cancer/colonandrectumcancer/detailedguide/colorectal-cancer-staged. Accessed 2015 Apr 14.

5. Stamos MJ, Murrell Z. Management of early rectal T1 and T2 cancers. Clin Cancer Res 2007; 13: 6885s-6889s.

6. Beynon J. An evaluation of the role of rectal endosonography in rectal cancer. Ann R Coll Surg Engl 1989; 71: 131-139.

7. Tapan Ü, Özbayrak M, Tatlı S. MRI in local staging of rectal cancer: an update. Diagn Interv Radiol 2014; 20: 390-398.

8. Watanabe H, Igari D, Tanahashi Y, Harada K, Saitoh M. Transrectal ultrasonotomography of the prostate. J Urol 1975; 114: 734-739.

9. Wild JJ, Reid JM. Diagnostic use of ultrasound. Br J Phys Med 1956; 11: 248-264.

10. Alzin HH, Kohlberger E, Schwaiger R, Alloussi S. Valeur de l'echographie endorectale dans la chirurgie du rectum. Ann Radiol 1983; 26: 334-336.

11. Dragsted J, Gammelgaard J. Endoluminal ultrasonic scanning in the evaluation of rectal cancer: a preliminary report of 13 cases. Gastrointest Radiol 1983; 8: 367-369.

12. Beynon J, Foy DM, Temple LN, Channer JL, Virjee J, Mortensen NJ. The endosonic appearances of normal colon and rectum. Dis Colon Rectum 1986; 28: 810-813.

13. Beynon J, Mortensen NJ, Foy DM, Channer JL, Virjee J, Goddard P. Pre-operative assessment of local invasion in rectal cancer: digital examination, endoluminal sonography or computed tomography? Br J Surg 1986; 73: 1015-1017.

14. Truong S, Hartung R, Rohrmann D. Preoperative staging and follow-up in rectal cancer using endorectal sonography. Zentralbl Chir 1986; 111: 333-338.

15. Rifkin MD, Wechsler RJ. A comparison of computed tomography and endorectal ultrasound in staging rectal cancer. Int J Colorectal Dis 1986; 1: 219-223.

16. Hildebrandt U, Feifel G. Preoperative staging of rectal cancer by intrarectal ultrasound. Dis Colon Rectum 1985; 28 : 42-46.

17. Badea R, Badea G, Philippi W, Dejica D, Bologa S, Cazacu $\mathrm{M}$. The value and limits of endorectal sonography in the preoperative stage classification of rectal cancer. Ultraschall Med 1988; 9: 265-269.

18. Dershaw DD. Endorectal sonography for rectal carcinoma. Bull N Y Acad Med 1992; 68: 411-419.

19. Badea R, Badea G, Dejica D, Henegar E. The role of transvaginal sonography as compared with endorectal sonography in the evaluation of rectal cancer: preliminary study. Surg Endosc 1991; 5: 89-91.

20. Scialpi M, Zottele F, Niccolini M, Dalla Palma F. Transvaginal echography in the study of the rectum. A new method with rectal distension with water. Radiol Med 1993; 85: 203-208.

21. Akbari RP, Wong WD. Endorectal ultrasound and the preoperative staging of rectal cancer. Scand J Surg 2003; 92 : 25-33. 
22. Hunerbein M, Dohmoto M, Haensch W, Schlag PM. Evaluation and biopsy of recurrent rectal cancer using three-dimensional endosonography. Dis Colon Rectum 1996; 39: 1373-1378.

23. Benacerraf BR, Benson CB, Abuhamad AZ, et al. Threeand 4-dimensional ultrasound in obstetrics and gynecology: proceedings of the American Institute of Ultrasound in Medicine Consensus Conference. J Ultrasound Med 2005; 24: 1587-1597.

24. Bamber J, Cosgrove D, Dietrich CF, et al. EFSUMB guidelines and recommendations on the clinical use of ultrasound elastography. Part 1: Basic principles and technology. U1traschall Med 2013; 34: 169-184.

25. Havre RF, Waage JR, Gilja OH, Odegaard S, Nesje LB. Real-Time Elastography: Strain Ratio Measurements Are Influenced by the Position of the Reference Area. Ultraschall Med 2011 Jun 10. doi: 10.1055/s-0031-1273247.

26. Waage JE, Havre RF, Odegaard S, Leh S, Eide GE, Baatrup G. Endorectal elastography in the evaluation of rectal tumours. Colorectal Dis 2011; 13: 1130-1137.

27. Cosgrove D, Piscaglia F, Bamber J, et al. EFSUMB guidelines and recommendations on the clinical use of ultrasound elastography. Part 2: Clinical applications. Ultraschall Med 2013; 34: 238-253.

28. Waage JE, Leh S, Rosler C, et al. Endorectal ultrasonography, strain elastography and MRI differentiation of rectal adenomas and adenocarcinomas. Colorectal Dis 2014 Nov 18. doi: 10.1111/codi.12845.

29. Zhuang H, Yang ZG, Wang ZQ, et al. Features of timeintensity curve parameters of colorectal adenocarcinomas evaluated by double-contrast enhanced ultrasonography: initial observation. Eur J Radiol 2012; 81: 677-682.

30. Lu M, Yan B, Song J, Ping W, Yue LX, Song B. Doublecontrast-enhanced sonography for diagnosis of rectal lesions with pathologic correlation. J Ultrasound Med 2014; 33: 575-583.

31. Wang Y, Li L, Wang YX, et al. Time-intensity curve parameters in rectal cancer measured using endorectal ultrasonography with sterile coupling gels filling the rectum: correlations with tumor angiogenesis and clinicopathological features. Biomed Res Int 2014; 2014: 587806.

32. Scialpi M, Niccolini M, Zottele F, Dalla Palma F, Scialpi P. A new method for study of the rectum using transvaginal ultrasound with water enema. Abdom Imaging 1996; 21: 342-344.

33. Scialpi M, Rotondo A, Angelelli G. Water enema transvaginal ultrasound for local staging of stenotic rectal carcinoma. Abdom Imaging 1999; 24: 132-136.

34. Sudakoff GS, Gasparaitis A, Michelassi F, Hurst R, Hoffmann K, Hackworth C. Endorectal color Doppler imaging of primary and recurrent rectal wall tumors: preliminary experience. AJR Am J Roentgenol 1996; 166: 55-61.

35. Alexander AA, Liu JB, Palazzo JP, et al. Endorectal color and duplex imaging of the normal rectal wall and rectal masses. J Ultrasound Med 1994; 13: 509-515.

36. Heneghan JP, Salem RR, Lange RC, Taylor KJ, Hammers LW. Transrectal sonography in staging rectal carcinoma: the role of gray-scale, color-flow, and Doppler imaging analysis. AJR Am J Roentgenol 1997; 169: 1247-1252.

37. Kwok H, Bissett IP, Hill GL. Preoperative staging of rectal cancer. Int J Colorectal Dis 2000; 15: 9-20.

38. Granero-Castro P, Muñoz E, Frasson M, et al. Evaluation of mesorectal fascia in mid and low anterior rectal cancer using endorectal ultrasound is feasible and reliable: a comparison with MRI findings. Dis Colon Rectum 2014; 57: 709-714.

39. Worrell S, Horvath K, Blakemore T, Flum D. Endorectal ultrasound detection of focal carcinoma within rectal adenomas. Am J Surg 2004; 187: 625-629.

40. Kim JC, Kim HC, Yu CS, et al. Efficacy of 3-dimensional endorectal ultrasonography compared with conventional ultrasonography and computed tomography in preoperative rectal cancer staging. Am J Surg 2006; 192: 89-97.

41. Hunerbein M, Pegios W, Rau B, Vogl TJ, Felix R, Schlag PM. Prospective comparison of endorectal ultrasound, three-dimensional endorectal ultrasound, and endorectal MRI in the preoperative evaluation of rectal tumors. Preliminary results. Surg Endosc 2000; 14: 1005-1009.

42. Glaser F, Layer G, Zuna I, van Kaick G, Schlag P, Herfarth C. Preoperative assessment of perirectal lymph nodes by ultrasound. Chirurg 1990; 61: 587-591.

43. Hildebrandt U, Feifel G. Importance of endoscopic ultrasonography staging for treatment of rectal cancer. Gastrointest Endosc Clin N Am 1995; 5: 843-849.

44. Kaur H, Choi H, You YN, et al. MR imaging for preoperative evaluation of primary rectal cancer: practical considerations. Radiographics 2012; 32: 389-409.

45. Park SH, Kim SJ, Kim EK, Kim MJ, Son EJ, Kwak JY. Interobserver agreement in assessing the sonographic and elastographic features of malignant thyroid nodules. AJR Am J Roentgenol 2009; 193: W416-W423.

46. Merino S, Arrazola J, Càrdenas A, et al. Utility and interobserver agreement of ultrasound elastography in the detection of malignant thyroid nodules in clinical care. Am J Neuroradiol 2011; 32: 2142-2148.

47. Ragazzoni F, Deandrea M, Mormile A, et al. High diagnostic accuracy and interobserver reliability of real-time elastography in the evaluation of thyroid nodules. Ultrasound Med Biol 2012; 38: 1154-1162.

48. Lim DJ, Luo S, Kim MH, Ko SH, Kim Y. Interobserver agreement and intraobserver reproducibility in thyroid ultrasound elastography. Am J Roentgenol 2012; 198: 896901.

49. Waage JE, Rafaelsen SR, Borley NR, et al. Strain Elastography Evaluation of Rectal Tumors: Inter- and Intraobserver Reproducibility. Ultraschall Med 2015 Apr 15. doi: 10.1055/s-0034-1398985.

50. Choi MY, Lee JW, Jang KJ. Distinction between benign and malignant causes of cervical, axillary, and inguinal lymphadenopathy: value of Doppler spectral waveform analysis. AJR Am J Roentgenol 1995; 165: 981-984.

51. Goh V, Padhani AR, Rasheed S. Functional imaging of colorectal cancer angiogenesis. Lancet Oncol 2007; 8: 245255. 